\title{
Was kann ein Pain Face Reader?
}

\author{
Wenn Patienten nicht in der Lage sind, Schmerzen zu verbalisieren, können mimische Veränderungen \\ wichtige Hinweise liefern. Bei der Entschlüsselung sollen künftig auch Computer helfen. Wie, das erklärt \\ Stefan Lautenbacher, Professor für physiologische Psychologie an der Universität Bamberg.
}

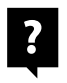

Prof. Dr. phil. Stefan Lautenbacher Universität Bamberg

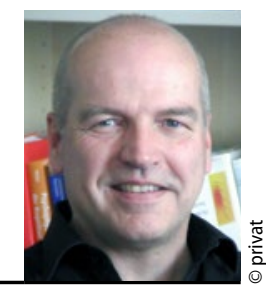

MMW: Die Mimik von Menschen, die Schmerzen haben, kann je nach Schmerzcharakter und Persönlichkeit sehr unterschiedlich ausfallen. Wie bringt man einen Computer dazu, trotzdem Schmerzen zu erkennen?

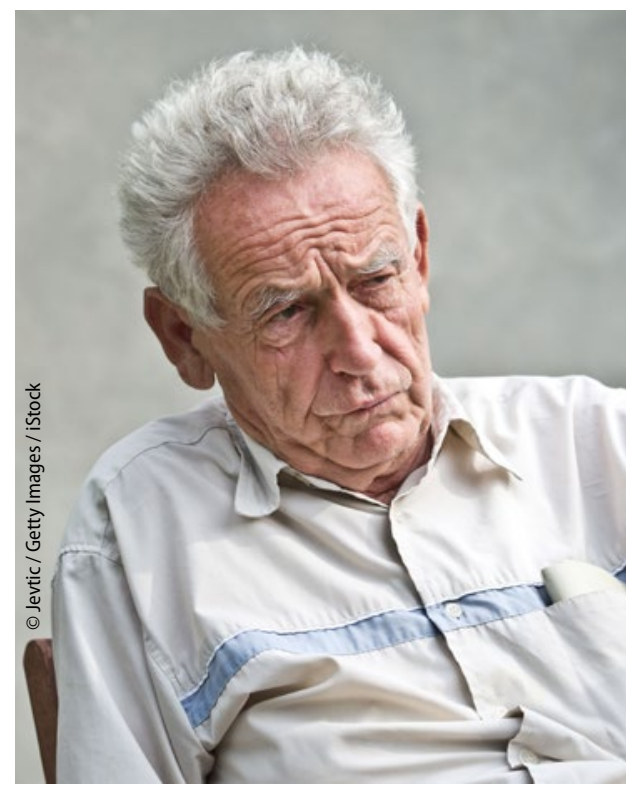

Fazies dolorosa: Ein Computer könnte helfen, wenn der Patient sich selbst nicht melden kann.
Lautenbacher: Die Vielfalt ist in der Tat ein Problem, aber es gibt auch eine Universalität: Bestimmte Bewegungen unseres Gesichtes drücken Schmerz aus, insbesondere die Intensität von Schmerz. Diese universellen Muster sind sogar interkulturell vergleichbar und auch schon bei Kleinstkindern vorhanden. Zusätzlich werden individuelle Ausdrucksformen erworben. Der Computer muss die Universalien kennen und er muss in der Lage sein, die verschiedenen individuellen Muster zu erkennen. So eine Aufgabe können Computer relativ gut lösen.

\section{MMW: Was sind das für universale} "Schmerzmuster", die der Computer erkennt?

Lautenbacher: Das Video-System von Fraunhofer, mit dem wir arbeiten, nutzt das von Physiologen entwickelte Facial Action Coding System. Dabei werden 44 Action Units detektiert. Ein Klassiker für Schmerz ist zum Beispiel die Action Unit 4, die Augenbrauen ziehen sich zusammen, und die Action Units 6 und 7, die Augenhöhlen verengen sich und die Backen kommen scheinbar ein bisschen nach oben. Weitere wichtige Hinweise sind die Action Units 9 und 10, die Oberlippe geht nach oben und die Nase bildet leichte Runzeln. Die mimischen Zeichen zeigen allerdings nur aktuelle Schmerzepisoden an.

MMW: Wie hoch ist die Trefferquote? Lautenbacher: Das hängt von der Anforderung ab. Bei einfachen Detektions- aufgaben im Offline-Bereich, wo Schmerz und Nichtschmerz unterschieden werden sollen, funktioniert das System schon ganz gut. Eine schwierige Aufgabe, die noch zu lösen ist: Veränderungen im Gesicht verursachen Falten, die zur Entdeckung von Action Units genutzt werden können. Gesichter von alten Menschen haben aber vorbestehende, nicht dynamische Falten. Bei ihnen mussten die Algorithmen angepasst werden, damit diese Falten nicht als Ausdrucksfalten interpretiert werden.

\section{MMW: Wo soll die automatische} Schmerzerkennung zum Einsatz kommen?

Lautenbacher: Das Haupteinsatzgebiet werden Kameras zur Schmerzdetektion in der postoperativen Phase sein. Kognitiv gesunde Menschen können Schmerzmittelpumpen kompetent bedienen, Demenzpatienten zum Beispiel können das nicht.

\section{MMW: Ersetzt der Computer dann} das Pflegepersonal?

Lautenbacher: Personell können wir das gar nicht leisten, einen Patienten, der nicht selbst einen Knopf drücken kann, nach einer Operation rund um die Uhr $\mathrm{zu}$ beobachten. Insofern ist so ein System eine große Hilfe. Wenn in der postoperativen Phase Schmerz auftritt, ist es doch toll, wenn dann ein Signal angeht, das einen menschlichen Entscheider herbeiholt, der sich ein Bild von dem $\mathrm{Pa}$ tienten macht.

Interview: Dr. Beate Schumacher 